\title{
Características clínico bioquímicas de pacientes con patología hipofisaria
}

\author{
D.A. DE LUIS, C. REINA \\ Instituto de Endocrinología y Nutrición. Facultad de Medicina. Valladolid. Unidad de \\ Apoyo a la Investigación. Sección de Endocrinología y Nutrición. Hospital Universitario \\ Río Hortega. Valladolid
}

\author{
CLINICAL AND BIOCHEMICAL CHARACTERISTICS OF PATIENTS \\ WITH PITUITARY DISEASE
}

\section{RESUMEN}

Objetivo: Los estudios descriptivos de patología hipofisaria en nuestro medio son escasos. Los adenomas pituitarios representan el 10-12\% de todas las neoplasias intracraneales. Son tumores cuyo origen se encuentra en las células de la adenohipófisis, dependiendo de la estirpe el comportamiento clínico y bioquímico es diferente. El objetivo de nuestro estudio fue la descripción de las características clínicas y bioquímicas de los pacientes con patología hipofisaria de nuestro centro.

Material y métodos: El estudio está basado en un análisis 58 pacientes con diagnóstico de tumor hipofisaria, en tratamiento y control periódico por la Sección de Endocrinología del Hospital Universitario Río Hortega de Valladolid entre los años 1999 y 2001. Se registraron los siguientes datos procedentes de la Historia Clínica de cada paciente: variables epidemiológicos, clínicos y analíticos.

Resultados: La edad media en el momento del diagnóstico fue 36,4 años (DS 16,34 años), representando la población femenina el 70,61\% del total. Los tumores más frecuentes fueron el prolactinoma y adenoma no funcionante siendo la amenorrea el motivo más frecuente de consulta al especialista $(15 \%)$ y destacando el médico de Atención Primaria como el facultativo que con mayor frecuencia establece la sospecha diagnóstica. La resonancia magnética nuclear inicial se realizó en 54 pacientes, de las cuales un $15,5 \%$ era normal, y el 78,6\% patológica, siendo los hallazgos más frecuentes los macro y microadenomas con una frecuencia ambos de $24,14 \%$. Las lesiones invasivas (craneofaringiomas, germinomas, adenomas invasivos) constituyen el 6,9\%. El síndrome depresivo, la hipertensión arterial y la diabetes mellitus en cualquiera de sus dos variantes destacaron como las patologías acompañantes más habituales en este tipo de pacientes.

Conclusiones: Nuestro estudio demostró como el prolactinoma es la patología hipofisaria más frecuente. La amenorrea, seguida de la galactorrea o la combinación de ambas es la clínica inicial más predominante. La respuesta al tratamiento médico y quirúrgico de los prolactinomas fue excelente.

PALABRAS CLAVE: Estudio descriptivo. Hipófisis. Tumores.

\section{ABSTRACT}

Objective: Descriptive studies in our country of hypophisis diseases are uncommon. Pituitary adenoma are unfrequent, however this pathology represent 10-12\% of brain tumors. Pituitary adenomas are derived of adenohypophisis cells, depending of cellular line, clinical and biochemical characteristics are diferents. The aim of our study was to describe these characteristics in our patients.

Material and methods: We study 58 patients with diagnosis of pituitary tumor, with regular follow-up in our Hospital, between 1999-2001. The next data were recorded: epidemiological, clinical and biochemical datas.

Results: Average age at diagnosis was 36.4 years (DS 16.34 years), with a $70.61 \%$ of females. The most frequent tumor was prolactinoma and non-functioning adenomas, amenorrhea was the most frequent symptom with the specialist physician $(15 \%)$ and the general practice physician was the first step in diagnosis protocol. Cerebral image technique was realized in 54 patients, with a $15.5 \%$ of normal exploration and $78.6 \%$ with lesions, with $24.14 \%$ of microadenoma and macroadenoma. Invasive lesions (craneopharingioms, germinomes and invasive adenomas) representing a 6.9\%. Depression, hipertension and diabetes mellitus were the most frequent diseases in these patients.

Conclusions: Prolactinoma is the most frequent hypophisis patology. Amenorrhea and/or galactorrhea are the most common symptom. Response to medical and surgical treatment was excelent in prolactinomas.

KEY WORDS: Descriptive study. Hyphofisis. Tumors.

De Luis DA, Reina C. Características clínico bioquímicas de pacientes con patología hipofisaria. An Med Interna (Madrid) 2005; 22: 364368.

\section{INTRODUCCIÓN}

Los adenomas pituitarios representan el $10-12 \%$ de todas las neoplasias intracraneales (1). Se trata de tumores cuyo ori- gen se encuentra en las células de la adenohipófisis. Su carácter histológico de benignidad no impide un comportamiento agresivo en relación con las alteraciones hormonales y compresión de estructuras del sistema nervioso central (2).

Trabajo aceptado: 11 de mayo de 2005

Correspondencia: D. A. de Luis. Director IEN. C/ Los Perales. 16. 47010 Valladolid. www.ienva.org 
Es significativa la ausencia de estudios descriptivos sobre esta patología en el ámbito sanitario español, la realización de este tipo de trabajos permitiría un aumento del conocimiento en la rápida sospecha diagnóstica inicial por parte de los distintos especialistas, protocolos de seguimiento, aproximación terapeútica y por último evaluación de los costes que todo ello representa.

El objetivo de nuestro estudio fue describir las características clínicas y bioquímicas de los pacientes con tumores hipofisarios de nuestro centro.

\section{MATERIAL Y MÉTODOS}

El estudio está basado en un análisis de 58 pacientes con diagnóstico de lesión hipofisaria, en tratamiento y control periódico por la Sección de Endocrinología del Hospital Universitario del Río Hortega de Valladolid entre los años 1999 y 2001. Se registraron los siguientes datos procedentes de la historia clínica de cada paciente:

1. Datos epidemiológicos: sexo, edad de diagnóstico, edad actual y procedencia (especialista que derivó al paciente a la Sección de Endocrinología).

2. Datos clínicos: clínica del debut, patología hipofisaria diagnosticada y patología acompañante. Otros datos recogidos fueron el peso, la talla, índice de masa corporal (IMC), tensión arterial sistólica y diastólica. El control del tumor se definió como la normalización de los niveles hormonales en el caso de los tumores funcionantes, así como la remisión del tamaño tumoral en el estudio por imagen.

3. Datos hormonales pre y post-tratamiento: se recogieron los valores de las siguientes hormonas en el momento inicial (previo a tratamiento) y los valores de las mismas en el último análisis de laboratorio de cada paciente: hormona estimulante tirotropa (TSH) (valor de referencia: 0,35-5 uUI/ml. Método ICMA, Abbott Park,IL, EE.UU.); tiroxina (T4Libre), (valor de referencia:0,68-1,5 ng/dl. Método ICMA, Abbott Park, IL, EE.UU.); hormona luteinizante (LH), (valor de referencia: hombres 1,8- 8,1 mUI/ml; mujeres, dependiendo de la fase del ciclo ovárico se establecen intervalos entre 1-92 $\mathrm{mUI} / \mathrm{ml}$. Método ICMA, Abbott Park, IL, EE.UU.); hormona estimulante folicular (FSH), (valor de referencia: hombres 1,3-13,3 $\mathrm{mUI} / \mathrm{ml}$; mujeres, como en el caso anterior entre 2-150 mUI/ml. Método ICMA,, Abbott Park, IL, EE.UU.); testosterona (valor de referencia: se establecen intervalos de edad para ambos sexos encontrando en los hombres rangos normales entre 2-1100 ng/dl y en las mujeres entre 2-110 ng/dl. Método ICMA, Abbott Park, IL, EE.UU.); estradiol (valor de referencia: hombres $25-107 \mathrm{pg} / \mathrm{ml}$, y en mujeres, según fase del ciclo ovárico se establecen intervalos entre $24-427 \mathrm{pg} / \mathrm{ml}$. Método MEIA, Abbott Park, IL, EE.UU.); progesterona (nivel de referencia: hombres entre0,10-0,20 ng/ml, en mujeres según fase del ciclo ovárico entre $0,10-15,90 \mathrm{ng} / \mathrm{ml}$. No tenemos ningún caso de mujer gestante, por lo que no incluimos los niveles normales en estas. Método ICMA, Abbott Park, IL, EE.UU.); Prolactina (nivel de referencia: hombres 2,6-18 ng/ml, mujeres 1,2-30 ng/ml. Método ICMA, Abbott Park, IL, EE.UU.); hormona gonadotropa (hGH), (valor de referencia: menor a $5 \mathrm{ng} / \mathrm{ml}$. Método inmunométrico, Llanberis, UK). Factor de crecimiento insulínico (IGF1) (valor de referencia: hombres adultos entre 100-591 ng/ml; mujeres adultas entre 96-502 ng/ml, a partir de los 39 años en ambos sexos la normalidad está en 0,78-303 ng/ml. Método RIA clásico, IGF1, Llanberis, UK). Cortisol plasma (valor de referencia: 6,033-30,15 ng/dl. Método FPIA, sistema TDX). Cortisol urinario (valor de referencia: 116-602 nmol/24 horas. Método FPIA, Sistema TDX). Hormona Estimulante Corticotropa (ACTH), (valor de referencia a las 8:00 horas: inferior a 60 $\mathrm{pg} / \mathrm{ml}$, Llanberis,UK ). Osmolaridad del plasma (valor de referencia: 275-295 mOsm/kg. Método del descenso crioscópico). Osmolaridad urinaria (valor de referencia: 300-900 $\mathrm{mmol} / \mathrm{kg}$. Método del descenso crioscópico).

Todos los valores de referencia que se citan hacen alusión a niveles normales en adultos y han sido realizados por el servicio de análisis clínicos del HURH.

4. Datos de imagen: se recogió el resultado de la resonancia magnética nuclear (RMN) en el momento inicial (previo a tratamiento), y el resultado de la última RMN realizada (posterior a tratamiento). Se empleó una intensidad de 0,5-1,5 Teslas o gauss con gadolinio.

Los tumores encontrados en la RMN se clasificaron en microadenomas si el tumor era menor de $10 \mathrm{~mm}$; macroadenoma si superaba los $10 \mathrm{~mm}$; adenomas invasivos si afectaban a senos cavernosos, seno esfenoidal, estructuras de la vía óptica, extensión a lóbulos cerebrales incluso oclusión del agujero de Monro; y otras lesiones: craneofaringiomas, germinomas, quiste aracnoideo y silla turca vacía.

5. Datos de valoración oftalmológica: El estudio campimétrico se realizó por el Servicio de Oftalmología de este hospital en aquellos pacientes que presentaban como parte de la clínica inicial déficits visuales o afectación de las estructuras del sistema nervioso central en la RMN. En la campimetría el médico explora la extensión del campo visual global del paciente mediante el examen de al menos ocho meridianos del campo visual (superior, inferior, nasal, temporal, nasal superior e inferior, y temporal superior e inferior) comparándolo con su propio campo visual. Para ello extiende el dedo índice de la mano y va desplazando esta de fuera hacia dentro, en cualquiera de los meridianos a explorar, a unos $50 \mathrm{~cm}$ del paciente, debiendo señalar este cuando comienza a ver el dedo índice del médico (la extensión, en grados, del campo visual normal aparece en la sección de neuro-oftalmología).

Se recogieron los resultados de las campimetrías, que se dividieron en normales y patológicas, clasificando a estas en hemianopsias, cuadrantanopsias, ceguera, otros déficits visuales y aquellas que no fueron valorables (por ausencia de colaboración del paciente) en el momento inicial (previo a tratamiento) y en la última prueba realizada (posterior al tratamiento).

6. Análisis estadístico: las variables cuantitativas se expresaron como media \pm desviación estándar (DS). Las variables cualitativas se expresaron como frecuencias. Las variables cuantitativas fueron comparadas con el test t-Student en variables paramétricas y la U-Mann en no paramétricas y las variables cualitativas se compararon con la Chi cuadrado. Se consideró significativo $\mathrm{p}<0,05$.

\section{RESULTADOS}

\section{CARACTERÍSTICAS CLÍNICO EPIDEMIOLÓGICAS}

La distribución por sexos fue varones $(29,31 \%)$ y mujeres $(70,69 \%)$, marcó una clara diferencia, en probable relación 
con el tumor hipofisario más frecuente, y de mayor prevalencia en mujeres jóvenes. El prolactinoma, como se muestra en la tabla I fue el más frecuente. En el capítulo de otros hallazgos se incluyeron (meningiomas, neurinomas, astrocitomas, hipofisis morfológicamente nromales con hiperprolactinemia, etc).

\section{TABLA I}

FRECUENCIA DE LOS SUBTIPOS DE TUMORES HIPOFISARIOS

\begin{tabular}{lcc}
\hline Patología hipofisaria & Frecuencia & Porcentaje \\
\hline Adenoma no funcionante & 13 & $22,4 \%$ \\
Prolactinoma & 28 & $48,3 \%$ \\
Acromegalia & 1 & $1,7 \%$ \\
Enfermedad de Cushing & 3 & $5,2 \%$ \\
Silla turca vacía & 4 & $6,9 \%$ \\
Craneofaringioma & 2 & $3,4 \%$ \\
Otros hallazgos & 7 & $12,1 \%$ \\
\hline Total & 58 & $100 \%$ \\
\hline
\end{tabular}

La edad media en el momento del diagnóstico fue 36,4 años (DS 16,34 años), con una edad mínima de presentación de 15 años y máxima de 82 años. El peso medio fue 67,25 kilogramos (DS $12,5 \mathrm{~kg}$ ) y un índice de masa corporal medio de 25,54 (DS 4,54). La media de años de seguimiento clínico de los pacientes fue de 61,68 meses (un mínimo de 1 mes y un máximo de 336 meses).

La tabla II refleja la sintomatología inicial por la que el paciente consulta al facultativo, en la que destaca la amenorrea como el motivo más frecuente $(15 \%)$. Los pacientes con dos o más síntomas asociados (no representados en la tabla) suponían el 10,2\% del total. EL 39,7\% de los casos fueron sospechados por el médico de Atención Primaria, el 20,7\% por el neurocirujano, el 13,8\% por el ginecólogo y el resto por otros facultativos.

\begin{tabular}{lcc}
\multicolumn{3}{c}{ TABLA II } \\
\multicolumn{1}{c}{ PATOLOGÍA INICIAL EN ORDEN DE FRECUENCIA } \\
\hline Síntoma inicial & $N^{\circ}$ absoluto & Porcentaje \\
\hline Amenorrea & 15 & $25,9 \%$ \\
Hirsutismo & 6 & $10,3 \%$ \\
Galactorrea & 5 & $8,6 \%$ \\
Amenorrea + galactorrea & 5 & $8,6 \%$ \\
Impotencia & 2 & $3,4 \%$ \\
Cefalea & 2 & $3,4 \%$ \\
Incremento de peso & 2 & $3,4 \%$ \\
Apoplejía hipofisaria & 2 & $3,4 \%$ \\
Cefalea + alteración visual & 2 & $3,4 \%$ \\
Alteración visual & 1 & $1,7 \%$ \\
\hline
\end{tabular}

\section{HALLAZGOS PATOLÓGICOS}

La tabla III muestra la media de las determinaciones hormonales en el momento del diagnóstico y en último análisis de laboratorio, tras el tratamiento médico y/o quirúrgico. En esta observamos que el valor hormonal que se modificó de una manera significativa fue la prolactina, tras el tratamiento con agonistas dopaminérgicos. La campimetría no se realizó en el 60,3\% de los casos, en los pacientes realizados se detectaron las siguientes alteraciones; hemianopsia en un 10,2\%, cuadrantanopsia en un $5,1 \%$, ceguera total en un $1,7 \%$, siendo normal en el resto de los casos.

La RMN mostró un 5\% de hipofisis normales y un $95 \%$ patológicas, siendo los hallazgos más frecuentes los macro y microadenomas con un frecuencia ambos de 24,13\%. Las lesiones invasivas (craneofaringiomas, germinomas, adenomas invasivos) constituyen el 7,9\%, seguidos de la imagen de la silla turca vacía con una frecuencia de $6,9 \%$.

Tras el tratamiento médico y/o quirúrgico, los resultados de la RMN Se modificaron significativamente, con los siguientes resultados; normal $29,31 \%$; microadenoma $15,5 \%$; macroadenomas y lesiones invasivas aparecían en un $3,44 \%$.

TABLA III

MEDIA DE VALORES HORMONALES PRE Y POSTRATAMIENTO

\begin{tabular}{|c|c|c|c|c|c|}
\hline Hormona & Media 1 & $S D$ & Media 2 & $S D$ & $p$ \\
\hline TSH uUI/ml & 1,870 & 1,26 & 3,373 & 13,15 & ns \\
\hline T4L ng/dl & 1,848 & 2,08 & 1,445 & 2,82 & ns \\
\hline $\mathrm{GH} \mathrm{ng} / \mathrm{ml}$ & 5,607 & 14,43 & 4,783 & 6,70 & ns \\
\hline IGF ng/ml & 17,397 & 23,76 & 18,669 & 15,68 & ns \\
\hline ACTH pg/ml & 26.831 & 19,52 & 54,338 & 39,20 & ns \\
\hline C. Sangre ng/ml & 18,578 & 15,11 & 15,946 & 10,93 & ns \\
\hline C. Orina nmol/24hs & 474,581 & 436,01 & 706,800 & 677,25 & ns \\
\hline $\mathrm{LH} \mathrm{mUl} / \mathrm{ml}$ & 9,508 & 10,88 & 8,207 & 10,45 & ns \\
\hline FSH mUl/ml & 9,675 & 14,22 & 9,076 & 14,64 & ns \\
\hline Estradiol pg/ml & 74,847 & 116,65 & 76,558 & 83,04 & ns \\
\hline Testosterona ng/ml & 32,255 & 86,26 & 244,348 & 262,94 & ns \\
\hline Progesterona $\mathrm{pg} / \mathrm{ml}$ & 1,670 & 4,22 & 0,870 & 1,55 & ns \\
\hline Prolactina ng/ml & 82,223 & 122,64 & 17,608 & 28,54 & $<0,05$ \\
\hline OSM.orina osm/L & 557,625 & 324,28 & 623,608 & 262,68 & ns \\
\hline OSM. Plasma Osm/L & 291,333 & 16,03 & 277,600 & 8,04 & ns \\
\hline
\end{tabular}

C. sangre: cortisol en plasma a las 8:00 horas. C. orina: cortisol en orina de 24 horas. Media 1: media de los valores hormonales en el momento del diagnóstico. Media 2: media de los valores hormonales en el último análisis de laboratorio realizado (post-tratamiento). 
De los 58 pacientes estudiados, 17 fueron sometidos a cirugía $(29,3 \%)$. El 70,7\% restante no recibieron ningún tipo de tratamiento ó tratamiento farmacológico. De los pacientes intervenidos quirúrgicamente, el 93,8\% se realizó por vía transesfenoidal, y el 6,3\% vía trasfrontal. Se utilizó radioterapia en un $13,8 \%$ de los casos y agonistas dopaminérgicos en un 48,3\%.

En los prolactinomas, el agonista dopaminérgico más empleado fue la cabergolina, en un $41,4 \%$ de los 28 prolactinomas se utilizó este fármaco, la bromocriptina se utilizó en un 6,9\% y en un $51,7 \%$ se aplicó cirugía. La dosis de cabergolina más empleada fue $1 \mathrm{mg}$ por semana, en un 24,1\% de los pacientes.

La normalización de los niveles de prolactina en los pacientes tratados con cabergolina sucedió en el 91,3\%. Esta normalización de los niveles ocurrió en el $80 \%$ de los pacientes tratados con bromocriptina y un $50 \%$ de los intervenidos

Concomitantemente se realizó el estudio de la patología acompañante que presentaban estos pacientes, obteniéndose los siguientes datos: 44,82\% no presentaban patología añadida; $24,13 \%$ presentaban una; $8,62 \%$ presentaban dos; y el $26,40 \%$ presentaban tres o más patologías asociadas al problema hipofisario. La tabla IV nos muestra cuales fueron los hallazgos más frecuentes, destacando en importancia el síndrome depresivo, la HTA y la diabetes mellitus (DM ) en cualquiera de sus dos variantes.

Por último se realizó un estudio sobre el consumo de recursos que precisan estos pacientes, únicamente desde el punto de vista de la lesión hipofisaria, recogiendo el número de RMN, perfiles hormonales y visitas al especialista que precisaron durante el seguimiento que recibieron en la consulta de Endocrinología (Tabla V).

\section{TABLA IV}

PATOLOGÍA ACOMPAÑANTE MÁS FRECUENTE

\begin{tabular}{lcc}
\hline Patología & Frecuencia & Porcentaje \\
\hline Bocio & 5 & $10,205 \%$ \\
DM & 7 & $14,285 \%$ \\
Otras alt. hormonales & 7 & $14,287 \%$ \\
Hipercolesterolemia & 4 & $8,163 \%$ \\
Hiperlipemia & 1 & $2,041 \%$ \\
Hiperuricemia & 1 & $2,041 \%$ \\
HTA & 9 & $18,367 \%$ \\
Sdr. depresivo & 7 & $14,28 \%$
\end{tabular}

TABLA V

RECURSOS EMPLEADOS

\begin{tabular}{llrc}
\hline Recurso & $N$ & Media & Desv.tipo \\
\hline RMN & 58 & 5,36 & 5,38 \\
Perfiles & 56 & 9,66 & 11,65 \\
Visitas & 57 & 11,05 & 13,13 \\
\hline
\end{tabular}

\section{DISCUSIÓN}

Nuestros resultados epidemiológicos son similares a los encontrados en otros trabajos. La distribución por edades y sexos en diferentes estudios establecen un pico de incidencia entre los 30 y 60 años, siendo más temprano en mujeres (2045 años) respecto a los varones (35-60 años) debido a la mayor presencia del prolactinoma, el tumor hipofisario más frecuente, entre las mujeres jóvenes (3). En nuestra muestra las mujeres representaban el 70,69\% del total.

En cuanto a los tumores más frecuentes, nuestros resultados son similares a los de otras series, mostrando como los adenomas no funcionantes y los prolactinomas como los más frecuentes. En el trabajo de Clayton y cols. (3) los prolactinomas aparecen con una frecuencia del 39\% definidos clínicamente, y del $27 \%$ en la demostración inmunohistoquímica; los adenomas no funcionantes en un $27 \%$ y a continuación los tumores productores de hGH en el 16\%. Según la edad poblacional estas frecuencias varían, encontrándose que en individuos menores de 20 años, la distribución es la siguiente: prolactinoma 85,9\%; tumor productor de ACTH 10,2\% y productor de hGH 2,6\% (4).

Los síntomas iniciales son debidos al exceso de producción de una hormona determinada (sobre todo prolactina y $\mathrm{GH})$ o al efecto masa que provocan en el SNC. No obstante en función de la serie estudiada los resultados pueden variar. De este modo sobre una serie de 684 pacientes tratados quirúrgicamente (2), se admite que la clínica hallada con más frecuencia fue la alteración del campo visual, mientras que en nuestra serie aparece en el 11,4\%, ya sea de forma aislada ó con otros síntomas acompañantes (sobre todo cefalea). La frecuencia de amenorrea, galactorrea o ambas se presentó en el 40,3\% de las mujeres (que representaban el $56,7 \%$ de la población), un $22,88 \%$ del total, y en nuestra serie aparecen en el 43,1\%. Estas diferencias se deben a que el estudio fue realizado por un Servicio de Neurocirugía, donde acuden los pacientes con lesiones más avanzadas. En otro trabajo sobre tumores pituitarios en ancianos (5) encontraron alteraciones visuales en el 40,2\%, hipopitituarismo en el 18,3\%, hiponatremia $7,3 \%$ y únicamente un $15,9 \%$ debutan con una clínica de exceso hormonal. La justificación la hallamos en que los tumores más frecuentes en ancianos son los no funcionantes (representan el tercer tumor más frecuente en esta etapa de la vida), con lo cual la clínica más habitual es la presentada por el efecto masa a nivel de la silla turca y el SNC.

No hemos encontrado ningún trabajo previo que analizará, el especialista que remite a los pacientes a la consulta del endocrino, quien es el especialista que establece la sospecha diagnóstica y solicita la realización de pruebas a su disposición. El 39,7\% de los pacientes son derivados al Servicio de Endocrinología por el médico de Atención Primaria con el fin de obtener la confirmación diagnóstica. En cambio, los Servicios de Ginecología y Neurocirugía derivan con el objetivo de una prescripción y dosis farmacológica más adecuada y mejor control de la patología en cuestión.

Aunque las alteraciones visuales son típicas de tumores no secretores (6), estas también pueden ser provocadas por el efecto masa del tumor secretor. Los datos de campimetría encontrados refieren una frecuencias para la hemianopsia bitemporal del 62\%, hemianopsia unilateral temporal del $26 \%$, cuadrantanopsia bitemporal del $14 \%$ y atrofia óptica ó disminución de la agudeza visual del $39 \%$. La diferencia en las alteraciones del campo visual con respecto a nuestro trabajo, pueden ser explicadas por el menor número de macroadenomas presentes en nuestro estudio. 
En los diferentes estudios realizados previamente, se ha analizado la relación entre los tumores hipofisarios y otras neoplasias de origen endocrino, la más frecuente es la existente entre tumores productores de GH y la neoplasia endocrina múltiple tipo I (7). En cambio no encontramos ningún dato sobre la relación con otras patologías, entre ellas alteraciones tiroideas. Estas suponen un aumento importante de la morbi-mortalidad (destaca el aumento de riesgo cardiovascular por la presencia significativa de hipertensión arterial, diabetes mellitus y dislipemias) y del coste socio-sanitario que todo ello supone, añadido al gasto que ya presente por el tratamiento, pruebas diagnósticas y de control (análisis de laboratorio, RMN, etc.) y seguimiento (visitas al especialista).

\section{Bibliografía}

1. Asa SL. The pathology off pituitary tumors. Endocrinol Metabol Clin North Am 1999; 28: 13-43.

2. Hakan Oruckaptan H, Ozcan Jenneusim. Pituitary Adenomas: Results of 684 surgically treated patients and review of the literature. Surg Neurol 2000; 53: 211-219.

3. Clayton RN. Sporadic pituitary tumours: from epidemiology to use of databases. Baillièrés Clinical Endocrinol Metabol 1999; 13: 451-460.

4. Flagin, G. Epidemiology and patogénesis of pituitary adenomas. Acta Endocrinologica 1993; 129 (Supl. 1): 1-5.

5. Turner HE. Pituitary tumours in the elderly. Baillièrés Clin Endocrinol
Hasta la fecha, todos los estudios realizados sobre este tema han sido retrospectivos (8), en la mayoría de las ocasiones con un número reducido de pacientes (9), y variables con valores sin estandarizar. Sería preciso corregir ambos factores para obtener un estudio con el suficiente poder estadístico y un buen diseño prospectivo para resolver cuestiones como la incidencia real, morbilidad, mortalidad, complicaciones, tratamiento adecuado, controles precisos y costes que todo ello origina.

En conclusión, nuestro estudio demostró como el prolactinoma es la patología tumoral hipofisaria más frecuente. La amenorrea, seguida de la galactorrea o la combinación de ambas es la clínica inicial más predominante. La respuesta al tratamiento médico y / o quirúrgico fue excelente.

Metabol 1997; 11: 407-422.

6. Freda PU, Post KD. Differential diagnosis of sellar masses. Advances in pituitary tumor therapy. Endocrinol Metabol Clin North Am 1999; 28: 81-112.

7. Ben-Shlomo A, Melmed S. Acromegaly Neuroendocrinology. Endocrinol Metab 2001; 30: 565-583.

8. Mascarell I, Camara R, Piñon F, Abad AL. Incidentalomas hipofisarios revisión de 21 casos. Endocrinología 2004; 51: 400-404.

9. Pascual Piazuelo MC, Bestrué M, Serrano Ponz M, López Gascón J. Estudio epidemiológico de adenomas hipofisarios en Aragón y La Rioja. Rev Neurol 2002; 34: 794-800. 